\title{
P04-09. Induction of cross-clade neutralizing antibodies with a prime/boost vaccine strategy focused on a neutralizing epitope
} S Zolla-Pazner*1, X Kong², T Cardozo², C Hioe ${ }^{2}$, S Cohen², X Jiang², MK Gorny ${ }^{2}, \mathrm{M} \mathrm{Totrov}^{3}$, A Pinter ${ }^{4}$, C Krachmarov ${ }^{4}, \mathrm{MS} \mathrm{Seaman}^{5}$, S Wang ${ }^{6}$ and $\mathrm{S} \mathrm{Lu}{ }^{6}$

Address: ${ }^{1}$ Pathology, NYU School of Medicine, New York, USA, ${ }^{2}$ New York University School of Medicine, New York, USA, ${ }^{3}$ Molsoft, LLC, La Jolla, CA, USA, ${ }^{4}$ Public Health Research Institute, Newark, USA, ${ }^{5}$ Beth Israel Deaconess Medical Center, Boston, MA, USA and ${ }^{6}$ University of Massachusetts Medical School, Worcester, MA, USA

* Corresponding author

from AIDS Vaccine 2009

Paris, France. 19-22 October 2009

Published: 22 October 2009

Retrovirology 2009, 6(Suppl 3):P37 doi:10.1 186/1742-4690-6-S3-P37

This abstract is available from: http://www.retrovirology.com/content/6/S3/P37

(c) 2009 Zolla-Pazner et al; licensee BioMed Central Ltd.

\section{Background}

Experiments were designed based on the hypothesis that recombinant vaccine constructs can focus the immune response on shared HIV-1 neutralizing epitopes, and that if not diverted by other biologically irrelevant epitopes, high titers of cross-clade neutralizing antibodies (ccNAbs) will be induced. Indeed, previous experiments showed that when the immune response was focused on V3, ccNAbs were induced (Zolla-Pazner et al, Virology, 2008).

\section{Methods}

A prime/boost regimen was used in which rabbits were primed $(3 \times)$ with clade C gp120 DNA and boosted $(2 \times)$ with: 1) a fusion protein in which the consensus clade $\mathrm{C}$ V3 sequence was fused to the C-terminus of MuLV gp70 (V3-gp70), or 2) the same V3 sequence was inserted into a structurally compatible site on cholera toxin B (V3CTB). Sera were tested for neutralizing activity in TZM-bl cells against a panel of primary isolates and a selection of Tier 1, Tier 2 clade B, and Tier 2 clade $C$ pseudoviruses (psVs) from the standard panel.

\section{Results}

Sera from rabbits boosted with V3-CTB neutralized four primary isolates from clades A, AG and B with higher 50\% neutralizing titers (NT50) than sera from V3-gp70boosted rabbits. For example, sera from all five V3-CTB rabbits neutralized Bx08 (with a geometric mean titer [GMT50] = 1:153) whereas only one of five V3-gp70 rabbit responded (1:11). Similarly, serum titers in response to V3-CTB were greater than those to V3-gp70 against 4/4 Tier 1 clade B and C psVs (GMT50 = 1:188 vs. 1:60 for V3gp70-immunized rabbits). Tier 2 clade $\mathrm{C}$ psVZM109F was also neutralized by sera from V3-CTB rabbits $($ GMT50 = $\sim 1: 20$ ).

\section{Conclusion}

A prime/boost vaccine regimen using gp120 DNA and V3scaffold protein immunogens induced ccNAbs. A newly designed V3-CTB protein boost induced the strongest ccNAb response. 